\title{
Thermodynamics of ligand binding to histone deacetylase like amidohydrolase from Bordetella/Alcaligenes
}

\author{
Christian Meyners ${ }^{a}$, Matthias G. J. Baud ${ }^{b, c}$, Matthew J. Fuchter ${ }^{b}$ \\ and Franz-Josef Meyer-Almes ${ }^{\mathrm{a} *}$
}

\begin{abstract}
Thermodynamic studies on ligand-protein binding have become increasingly important in the process of drug design. In combination with structural data and molecular dynamics simulations, thermodynamic studies provide relevant information about the mode of interaction between compounds and their target proteins and therefore build a sound basis for further drug optimization. Using the example of histone deacetylases (HDACs), particularly the histone deacetylase like amidohydrolase (HDAH) from Bordetella/Alcaligenes, a novel sensitive competitive fluorescence resonance energy transfer-based binding assay was developed and the thermodynamics of interaction of both fluorescent ligands and inhibitors to histone deacetylase like amidohydrolase were investigated. The assay consumes only small amounts of valuable target proteins and is suitable for fast kinetic and mechanistic studies as well as high throughput screening applications. Binding affinity increased with increasing length of aliphatic spacers $(n=4-7)$ between the hydroxamate moiety and the dansyl head group of ligand probes. Van't Hoff plots revealed an optimum in enthalpy contribution to the free energy of binding for the dansyl-ligand with hexyl spacer. The selectivity in the series of dansyl-ligands against human class I HDAC1 but not class II HDACs 4 and 6 increased with the ratio of $\Delta \mathbf{H}^{0} / \Delta \mathrm{G}^{0}$. The data clearly emphasize the importance of thermodynamic signatures as useful general guidance for the optimization of ligands or rational drug design. Copyright $\odot 2014$ John Wiley \& Sons, Ltd.

Additional supporting information may be found in the online version of this article at the publisher's web site.
\end{abstract}

Keywords: histone deacetylase; HDAC; thermodynamic signatures; ligand binding; inhibitors

\section{INTRODUCTION}

Thermodynamic studies on ligand-protein binding have become increasingly important in the process of drug design in recent years (Chaires, 2008; Ladbury et al., 2009; Kawasaki and Freire, 2011).

Understanding the nature of molecular interactions requires structural studies and a thorough thermodynamic dissection of the forces that drive the recognition process. Molecular recognition through non-covalent binding includes both enthalpic and entropic contributions, with such binding processes governed by hydrogen bonding, metal coordination, hydrophobic interactions, and other less frequent interactions like halogen bonds or cation- $\pi$-interactions as well as solvation effects and dynamic structural changes of interacting ligand and protein (Chaires, 2008; Bissantz et al., 2010; Bronowska, 2011). In general, a negative free energy of binding $\left(\Delta G^{0}\right)$ indicates a spontaneous process and in the case of ligand-protein binding defines the ratio of bound complex concentration and the concentrations of free binding partners at chemical equilibrium. Consequently, $\Delta G^{0}$ values can be calculated from dissociation constants $\left(K_{d}\right)$ according to

$$
\Delta G^{0}=R T \ln \left(K_{d}\right)
$$

Furthermore, $\Delta G^{0}$ is composed of an enthalpic term $\left(\Delta H^{0}\right)$ and an entropic term $\left(-\mathrm{T} \Delta \mathrm{S}^{0}\right)$ at constant pressure:

$$
\Delta G^{0}=\Delta H^{0}-T \Delta S^{0}
$$

The balance between enthalpic and entropic components is called the thermodynamic signature of a ligand or drug with respect to its target protein. Traditionally, most drugs have been optimized with respect to affinity and therefore binding free

\footnotetext{
* Correspondence to: Franz-Josef Meyer-Almes, University of Applied Sciences Darmstadt, Schnittspahnstr. 12, Darmstadt, 64287, Germany.

E-mail: franz-josef.meyer-almes@h-da.de

a C. Meyners, F.-J. Meyer-Almes

Department of Chemical Engineering and Biotechnology, University of Applied Sciences Darmstadt, Darmstadt 64287, Germany

b M. G. J. Baud, M. J. Fuchter

Department of Chemistry, Imperial College London, London SW7 2AZ, United Kingdom

c M. G. J. Baud

Department of Chemistry, University of Cambridge, Lensfield Rd, CB2 1EW, United Kingdom
}

Abbreviations: $A M C$, 7-amino-4-methylcoumarin; EDCl, 1-ethyl-3-(3dimethylaminopropyl)carbodiimide; Dansyl, 5-(dimethylamino)naphthalene1-sulfonyl; FRET, fluorescence resonance energy transfer; HDAH, histone deacetylase like amidohydrolase from Bordetelle/Alcaligenes; HDAC, histone deacetylase; $K_{d}$-value, dissociation constant; $S A H A$, suberoylanilide hydroxamic acid; MB275, 4-[3-(3-bromo-4-hydroxy-phenyl)-2-(N-hydroxyimino) propanamido]-N-hydroxybutanamide. 
energy. This practice has been recently highlighted by Stahl et al. who claimed that "optimizing for free energy is still the only viable approach to structure-based design" because enthalpic and entropic contributions to binding depend highly on the interaction system under investigation (Bissantz et al., 2010). However, it is well known that over the course of traditional lead optimization, improvements in compound potency through the addition of hydrophobic moieties to the core of the lead structure usually result from an entropic contribution to binding. Such an approach has serious drawbacks, including unfavorable effects on the physiochemical properties of the ligand and a loss in drug selectivity, because binding is principally driven by the non-specific hydrophobic effect. On the other hand, the design of optimal non-covalent interactions between a ligand and a protein to improve $\Delta \mathrm{H}^{0}$ is exceptionally difficult. Ladbury et al. (2009) have highlighted the value of enthalpy data for aiding lead structure optimization. The authors exemplified their approach through retroperspective analysis of the development of the statin class of drugs, which inhibit the enzyme 3-hydroxy3-methylglutaryl coenzyme A reductase, and through analysis of HIV protease inhibitors. In both cases, best-in-class compounds were associated with improved $\Delta \mathrm{H}^{0}$ (more negative) contributions. The value of enthalpy-driven optimization was also demonstrated by studies on inhibitors of plasmepsin II, a hemoglobin-degrading enzyme that is a key component in the life cycle of the Plasmodium parasites responsible for malaria (Ruben et al., 2006). Plotting the binding constants of 71 inhibitors versus the ratio $\Delta H^{0} / \Delta G^{0}$ revealed a so-called enthalpy funnel, in which the enthalpy/entropy combinations narrowed as affinity increased. The optimal balance in this particular molecular system arose when the free energy partitioned to an approximate one third enthalpic and two thirds entropic contribution. The heat of interaction $\left(\Delta \mathrm{H}^{0}\right)$ can be either directly measured using isothermal titration calorimetry or calculated from linear fits to binding free energy data as a function of temperature according to equation 2 . The assumption that $\Delta \mathrm{H}^{0}$ is constant over relatively small ranges of temperatures, for example, $10-20 \mathrm{~K}$, is justified in many protein-ligand systems. Another important aspect in interpreting enthalpic and entropic contributions to $\Delta \mathrm{G}^{0}$ is the molecular dynamics (MD) of flexible protein-ligand complexes. Non-covalent binding can be positively cooperative, for example, if the structure of the target protein is tightened upon the formation of additional interactions, (Williams et al., 2003) with bonding opposing motion and vice versa. Therefore, in combination with structural data and MD simulations, thermodynamic studies are supposed to provide relevant information about the mode of interaction between compounds and target proteins.

In this study, we investigated the thermodynamics of ligand binding to HDAH from Bordetella/Alcaligenes (Hildmann et al., 2004). HDAH is structurally closely related to human HDACs (Nielsen et al., 2005). HDAH and the second catalytic domain of human HDAC6 share an identity of $35 \%$. Indeed, a large amount of data has been accumulated that prove that HDAH serves as a good model for HDACs, particularly for HDAC6. This fact is among others reflected by the similar selectivities for substrates and inhibitors of these enzymes (Hildmann et al., 2006; Riester et al., 2009). HDACs are widely distributed and differently expressed in different cell types. There are 18 isoforms of human HDACs that are divided into three classes of zinc cation dependent enzymes and one class, the sirtuins, which require $\mathrm{NAD}^{+}$as coenzyme for their activity. Class I consist of isoforms HDAC1, HDAC2, HDAC3, and HDAC8; class II is subdivided into class lla including HDAC4, HDAC5, HDAC7, and HDAC9, which shuttle between nucleus and cytoplasm in a highly regulated way, and class Ilb enzymes HDAC6 and HDAC10, which in contrast to all other HDACs contain two catalytic domains. Class Ila and class Ilb exhibit completely different substrate specificities, with class IIb HDACs having a preference for cytosolic substrates, for example, $\alpha$-tubulin, HSP90, or cortactin for HDAC6 (Aldana-Masangkay and Sakamoto, 2011). Based on multiple sequence alignments, HDAC11 was assigned to class IV. HDACs and their antagonistic opponents, histone acetyl transferases, play pivotal roles in cellular differentiation, proliferation, apoptosis, and the pathogenesis of malignant diseases through balancing the acetylation status of histones and many non-histone proteins (Ropero and Esteller, 2007; Marks, 2010). While initially, these enzymes were only recognized to deacetylate $\mathrm{N}$-terminal lysine residues of histones, they were subsequently identified to have many cytosolic substrates, underlining the broad functionality of HDACs in controlling many cellular processes (Peng and Seto, 2011). A wealth of experimental data has been collected over the last decade proving HDACs to be highly relevant cancer targets (Ropero and Esteller, 2007; Shankar and Srivastava, 2008). Consequently, much effort has been invested in identifying HDAC inhibitors and their subsequent development into drugs against certain types of cancer. The rather unselective SAHA (commercial name ZOLINZA by Merck \& Co.) and the cyclic and disulfide bridged romidepsin (commercial name Istodax by Celgene) have been approved by the US Food and Drug Administration as first-in-class drugs against cutaneous T-cell lymphoma, via the inhibition of HDACs. There are currently more than 130 open clinical trials involving HDAC inhibitory drugs in the treatment of numerous forms of cancer (Clinical Trials Website 2013). Recent efforts in this area have become focused on the development of isoform selective HDAC inhibitors, which are hypothesized to have improved efficacy and to cause fewer side effects like fatigue, nausea, dehydration, diarrhea, thrombocytopenia, or electrocardiogram changes (Marks, 2010).

To enable detailed thermodynamic studies on the interaction of small organic inhibitors to HDAH, a series of dansylconjugate ligands with alkyl-chains of different length have been synthesized and used to develop FRET-based binding assays for HDAH. The binding data obtained were of excellent quality and were allowed for the calculation of $\Delta G^{0}, \Delta H^{0}$, and $\Delta S^{0}$ of the binding event. To complement this binding data, the molecular interactions and the structural flexibility of the complexes were further investigated using MD simulations. The selectivity of all ligands was measured in enzyme activity assays against a panel of recombinant human HDACs. The association and dissociation kinetics of dansyl-ligand binding to $\mathrm{HDAH}$ were also measured to demonstrate the feasibility of the described assay for the investigation of fast binding reactions and getting insight in reaction mechanisms.

\section{MATERIALS AND METHODS}

\section{Materials}

All chemicals if not stated otherwise were obtained from Sigma/Aldrich (USA). SAHA was purchased from Cayman Chemical (USA), and MB275 was prepared according to Fuchter 
et al. (2012) (referring to compound 25 of that publication). The synthesis including chemical analytics of the six dansylconjugates is described in the supporting information. Histagged FB188 HDAH was prepared as described elsewhere (Hildmann et al., 2004). The assay buffer consisted of $250 \mathrm{mM}$ sodium chloride, $250 \mu \mathrm{M}$ ethylenediaminetetraacetic acid, $15 \mathrm{mM}$ Tris- $\mathrm{HCl}, 0.001 \%$ Pluronic $\mathrm{F}-127$ and $50 \mathrm{mM}$ potassium phosphate at $\mathrm{pH}$ 8.0. The buffer components were purchased from Merck (Germany), Roth (Germany), and Sigma-Aldrich (USA). DMSO, tris(2-carboxyethyl)phosphine, and trypsin (bovine pancreas) were purchased from Sigma-Aldrich, and Boc-Lys(Ac)-AMC and Boc-Lys(trifluoacetyl)-AMC from Bachem (Switzerland). The recombinant human histone deacetylases were obtained from BPS Bioscience (USA). All reactions were performed in black half area 96-well microplates (Greiner bioone, Germany).

\section{Fluorescence resonance energy transfer-based binding assay}

To investigate the direct binding of the dansyl-conjugate probes $\mathbf{5 a - f}$ to HDAH $500 \mu \mathrm{l}$ of a $0.1 \mu \mathrm{M}$ HDAH solution in assay buffer $\mathrm{pH} 8.0$ was filled in a cuvette and equilibrated to the desired final temperature in a spectrofluorimeter (Hitachi, F-7000). After equilibration, the concentration of the corresponding dansyl-conjugate was increased successively by adding small volumes of pre-equilibrated probe solved in assay buffer. After each addition, the time course of the FRET signal from the intrinsic tryptophans (donor) of $\mathrm{HDAH}$ to the dansyl moiety (acceptor) of the probe was measured at an excitation wavelength of $285 \mathrm{~nm}$ (slit $5 \mathrm{~nm}$ ) and an emission wavelength of $525 \mathrm{~nm}$ (slit $10 \mathrm{~nm}$ ) until the mean signal changed less than $1 \%$ over a period of $60 \mathrm{~s}$. To determine the contribution of directly excited ligand to the fluorescence signal at $525 \mathrm{~nm}$, a titration experiment in the absence of HDAH was performed in parallel. The fluorescence signal of the corresponding dansyl-conjugate at each concentration was substracted from the fluorescence signal of its mixture with HDAH and then normalized with respect to maximal binding. The competitive binding assay was performed as follows. At first, a protein-ligand complex was pre-formed by mixing $1.0 \mu \mathrm{M}$ 5e and $100 \mathrm{nM} \mathrm{HDAH}$ and incubating the mixture at the desired temperature until the mean FRET signal (fluorescence intensity at $525 \mathrm{~nm}$ ) remained constant within an error of less than $1 \%$ over a period of $60 \mathrm{~s}$. Then increasing amounts of compounds were added. Again, it was assumed that chemical equilibrium was achieved when the change of FRET signal was less than $1 \%$. The contribution of $\mathbf{5 e}$ and the respective compound to the fluorescence intensity was determined in a parallel titration experiment in the absence of $\mathrm{HDAH}$, subsequently subtracted from the fluorescence intensities in the presence of HDAH and normalized as described before in the direct binding mode.

\section{Calculation of binding constant $K_{d}$}

The dissociation constants, $K_{d}$-values, of the dansyl-conjugates with HDAH were determined from titrations of the protein with the corresponding conjugate measuring direct binding and exploiting the change in FRET signal (intensity at $525 \mathrm{~nm}$ ). The binding degree, $\mathrm{BD}$, was calculated from the FRET signal, $F$, of each data point, which was corrected for background signal and normalized to the maximal FRET signal, $F_{\text {max }}$, and then fitted to the following model function for ligand binding using GraphPad Prism (GraphPad Software, USA) revealing the corresponding $\mathrm{K}_{\mathrm{d}}$-value:

$$
\begin{aligned}
B D & =\frac{F}{F_{\max }} \\
& =\frac{0.5}{[E]_{0}}\left\{[L]_{0}+[E]_{0}+K_{d}-\sqrt{\left([L]_{0}+[E]_{0}+K_{d}\right)^{2}-4[E]_{0}[L]_{0}}\right\}
\end{aligned}
$$

where $K_{d}$ is the dissociation constant, $[L]_{0}$ is the total concentration of the respective dansyl conjugate, and $[E]_{0}$ denotes the total concentration of HDAH.

The $K_{d}$-values of compounds being potential binders to the protein were determined using the competition binding assay. In these cases, the dansyl-ligand $\mathbf{5 e}$ was used as fluorescent ligand probe and its dissociation constant, $K_{1}$, as determined from direct binding experiments at the respective temperature was used to calculate the $K_{d}$-values of the compounds, $K_{2}$, by fitting the data to the following model function using GraphPad Prism (GraphPad Software, USA):

$$
\frac{F}{F_{\max }}=\frac{1}{2[E]_{0}}\left(-p+\sqrt{p^{2}+\frac{4[E]_{0} K_{2}[L]_{0}}{K_{1}\left(\frac{K_{1}}{[L]_{0}}+1\right)}}\right),
$$

With

$$
p=\frac{K_{2}+\frac{K_{1}}{[L]_{0}}\left(K_{2}+[I]_{0}-[E]_{0}\right)}{\frac{K_{1}}{[L]_{0}}\left(\frac{K_{1}}{[L]_{0}}+1\right)},
$$

where the probe concentration, $[L]_{0}$, was held fix, $[E]_{0}$ was the total protein concentration, and $\left[I_{0}\right.$ was the compound concentration, which was varied. It is assumed that the free concentration of the probe remains unchanged upon binding to $\operatorname{HDAH}\left([L]=[L]_{0}\right)$. This condition is essentially fulfilled because of the 10 -fold excess of the probe over HDAH. The derivation of equation 4 is given in the supporting information. It is to mention that $K_{1}$ of the starting complex should be in the same order than the $K_{d}$ value of the compound to be calculated. Compounds with $K_{d}<<K_{1}$ can only be recognized qualitatively as tight binders. But a calculation of $\mathrm{K}_{\mathrm{d}}$ values is not possible because of stoichiometric displacement of the probe upon the addition of increasing amounts of compound. If $K_{d}>>K_{1}$, solubility problems or strong autofluorescence or quenching of test compounds may limit the use of high compound concentrations, which must be applied to displace the probe from HDAH. However, although the quantification of $K_{d}$ values is not possible in cases of extreme $K_{d} / K_{1}$ ratios, the compounds can easily be classified as strong or weak/non-binders.

\section{Calculation of thermodynamic parameters $\Delta H^{0}, \Delta S^{0}$, and $\Delta G^{0}$}

The $K_{d}$ values of a ligand at eight different temperatures from 18 to $37^{\circ} \mathrm{C}$ were transformed in binding free energy, $\Delta G^{0}$, according to equation 1. The data of van't Hoff plots $\left(\Delta G^{0}\right.$ versus temperature) were fitted to equation 2 using GraphPad Prism (GraphPad Software, USA) and yielding the desired changes in enthalpy, $\Delta H^{0}$, and entropy, $\Delta S^{0}$. 


\section{Binding kinetics}

All association and dissociation kinetics were followed using a spectrofluorometer (Hitachi, F-7000). Fluorescence was excited at $285 \mathrm{~nm}$, and the change in fluorescence intensity at $525 \mathrm{~nm}$ was measured as a function of time. The association kinetics of $\mathrm{HDAH}$ and dansyl-ligands were initiated by rapid mixing of both reactands at the denoted concentrations in assay buffer at $21^{\circ} \mathrm{C}$. To obtain the dissociation rates of the dansyl-ligands, a high molar excess of $500 \mu \mathrm{M}$ non-fluorescent SAHA was added to a preformed complex consisting of $200 \mathrm{nM} \mathrm{HDAH}$ and one of the respective dansyl-ligands $(12.8 \mu \mathrm{M} \mathrm{5c}, 6.4 \mu \mathrm{M} \mathbf{5 d}, 1.6 \mu \mathrm{M} \mathbf{5 e}$, or $1.6 \mu \mathrm{M} \mathbf{5 f}$ ) in assay buffer at $21^{\circ} \mathrm{C}$, and the corresponding displacement kinetics were measured as described.

\section{Enzyme activity assays}

The inhibitory potential of dansyl-ligands, MB275, and SAHA against recombinant human HDACs was determined using fluorogenic enzyme activity assays according to Wegener et al. (2003). In short, the ligands were pre-incubated with HDAC to allow for interaction. The addition of substrate (Boc-Lys(Ac)-AMC for HDACs 1, 6 and Boc-Lys(trifluoracetyl)-AMC for HDACs 4, 8) started the enzyme reaction. After $60 \mathrm{~min}$, the reaction was stopped, and the deacetylated substrate was converted in a fluorescent product using trypsin. The released AMC served as a measure of enzyme activity. In contrast, HDAH assays were performed in one step by combining the addition of substrate and trypsin. This became possible because of the insensitivity of HDAH against trypsin digestion.

\section{Docking and molecular dynamics simulations}

Several X-ray structures of HDAH are available (2VCG, 2GH6, $1 Z Z 0,1 Z Z 1$, and $1 Z Z 3)$. The structure of a complex between $\mathrm{HDAH}$ and SAHA (1ZZ1) was selected because of its high resolution of $1.57 \AA$. Accordingly, computational docking studies were carried out on the dansyl-conjugates $\mathbf{5 a - f}$ and all compounds by using SwissDock (http://www.swissdock.ch/) (Grosdidier et al., 2011) in the accurate mode and allowing the side chains to be flexible within $5 \AA$ of any atom of the corresponding ligand in its reference binding mode. Docking was performed on the protein structure (PDB 1ZZ1) in Protein Data Bank (PDB) and the ligand in Tripos Mol2 format. The best poses of ligand-protein complex structures were selected for MD-simulations using Gromacs V. 4.0.7.21 (Hess et al., 2008) with an implemented AMBER-99 forcefield (DePaul et al., 2010). The particle mesh Ewald method was used for the treatments of long-range electrostatic interactions. The enzyme-ligand complexes were subjected to two steps of energy minimization in vacuo using steepest descent and conjugate gradient method to remove bad van-der-Waals (vdW) contacts. Then the protein was solvated in a periodic cubic box of Tip4 water molecules (Jorgensen and Madura, 1985) containing $\mathrm{Na}^{+}$and $\mathrm{Cl}^{-}$ions corresponding to a final concentration of $250 \mathrm{mM}$ $\mathrm{NaCl}$, which complied with the salt concentration of the assay buffer employed. Approximately 35,000 water molecules were added to each system. After another energy minimization of the solvated complex position-restrained dynamics simulations were run to soak the water into the enzyme-ligand complex ( $200 \mathrm{ps}$ at $100 \mathrm{~K}, 60 \mathrm{ps}$ at $200 \mathrm{~K}$, and $200 \mathrm{ps}$ at $300 \mathrm{~K}$ ) followed by an equilibration period of unconstrained $2 \mathrm{~ns}$ MD-simulation at $300 \mathrm{~K}$. Finally, two productive MD simulations were performed both over a period of $20 \mathrm{~ns}$ at the same temperature. The trajectory traces were visualized and analyzed using the program VMD 1.8.7.24 (University of Illinois). Non-polar and polar solvent accessible surface areas have been calculated for the binding pocket using the implemented Shrake-Rupley algorithm (Shrake and Rupley, 1973) and a probe radius of $1.4 \AA$. The binding pocket was defined to consist of amino acids $21,98,100,140,142,143$, 151, 152, 153, 180, 182, 207, 208, 268, 275, 309, 310, and 312. In addition, root mean square fluctuation (RMSF) values as well as root-mean-square distance curves were calculated in VMD. A water distribution map was generated by measuring the distance between each water-oxygen atom and both, the hydroxyl-oxygen of Y312 and the $C \zeta$-atom of F208 in 2000 snap shots using VMD and creating two-dimensional histograms by counting the occurrence of water oxygen atoms in a two-dimensional distance grid with $0.1 \AA$ A bins using MatLab (Mathworks Inc., USA).

\section{RESULTS AND DISCUSSION}

\section{Design and synthesis of histone deacetylase like amidohydrolase probes}

For the development of a FRET-based assay, HDAH probes were designed according to the accepted HDACi pharmacophore (Zhang et al., 2011) consisting of a zinc-chelating hydroxamate group, an alkyl spacer with varying chain length and a dansyl moiety as the capping group. The dansyl moiety was selected to serve as acceptor dye for the fluorescence of intrinsic tryptophan residues (donor) of HDAH. It was also assumed that the relatively small aromatic dansyl moiety, which contained a sulphonamide linkage to the binding moiety would be a suitable substitute for an aromatic capping group, a key feature of many inhibitors of HDAH and other HDACs (Riester et al., 2007; Riester et al., 2009). The synthesis of the dansyl-hydroxamic acid conjugates was straightforward (Figure 1). Condensation between the commercially available dansyl chloride $\mathbf{1}$ and aminoacids 2a-f under basic conditions afforded sulphonamides $\mathbf{3 a - f}$ in good to excellent yield (58-85\%). Further EDCI mediated coupling with benzylhydroxylamine afforded hydroxamic acid precursors $\mathbf{4 a - f}$ in moderate to excellent yield (48-92\%). Final hydrogenolysis of the benzyl protecting group afforded dansyl-conjugate probes $\mathbf{5 a - f}$ in $57-85 \%$ yields. See the supporting infomation for detailed reaction conditions and analytical data.

\section{Isothermal binding of dansyl-conjugates and non-fluorescent inhibitors to histone deacetylase like amidohydrolase}

Binding of the dansyl-conjugates to HDAH resulted in a pronounced FRET from intrinsic tryptophan residues of the protein to the chromophore moiety of the dansyl-ligand probes. This was clearly indicated by a decrease of the tryptophan emission and a simultaneous increase in dansyl fluorescence intensity (Figure 2). The increase in fluorescence intensity at $525 \mathrm{~nm}$ was exploited to obtain binding isotherms at eight different temperatures between 18 and $37^{\circ} \mathrm{C}$ with a precision of $\pm 0.1^{\circ} \mathrm{C}$. The dansyl-conjugate $\mathbf{5 f}$ with highest affinity was used to determine the binding stoichiometry in a titration using concentrations much higher than the corresponding $K_{d}$ value of $60 \mathrm{nM}$. The ratio between $\mathbf{5 f}$ and HDAH was proven to be 1:1 (Figure S5). The same stoichiometry was assumed for complexes between $\mathrm{HDAH}$ and the other ligands with similar structure. Dansyl-conjugates $\mathbf{5 a}$ and $\mathbf{5 b}$ only exhibited weak binding to HDAH. Ligand $\mathbf{5 b}$ 

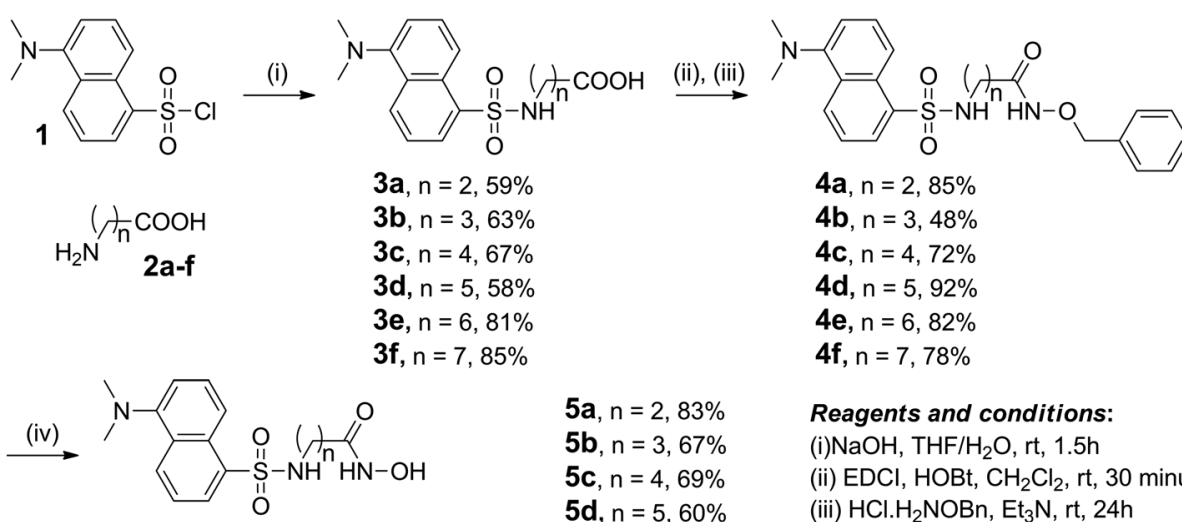

$5 \mathbf{a}, \mathrm{n}=2,83 \%$

$5 b, n=3,67 \%$

5c, $n=4,69 \%$

$\mathbf{5 d}, \mathrm{n}=5,60 \%$

5e, $n=6,85 \%$

$\mathbf{5 f}, \mathrm{n}=7,57 \%$

Reagents and conditions:

(i) $\mathrm{NaOH}, \mathrm{THF} / \mathrm{H}_{2} \mathrm{O}, \mathrm{rt}, 1.5 \mathrm{~h}$

(ii) $\mathrm{EDCl}, \mathrm{HOBt}, \mathrm{CH}_{2} \mathrm{Cl}_{2}, \mathrm{rt}, 30$ minutes

(iii) $\mathrm{HCl} . \mathrm{H}_{2} \mathrm{NOBn}, \mathrm{Et}_{3} \mathrm{~N}, \mathrm{rt}, 24 \mathrm{~h}$

(iv) $\mathrm{H}_{2}, \mathrm{Pd} / \mathrm{C}, \mathrm{MeOH}, \mathrm{rt}, 3 \mathrm{~h}$

Figure 1. Synthesis scheme of dansyl-conjugate probes for HDAH.

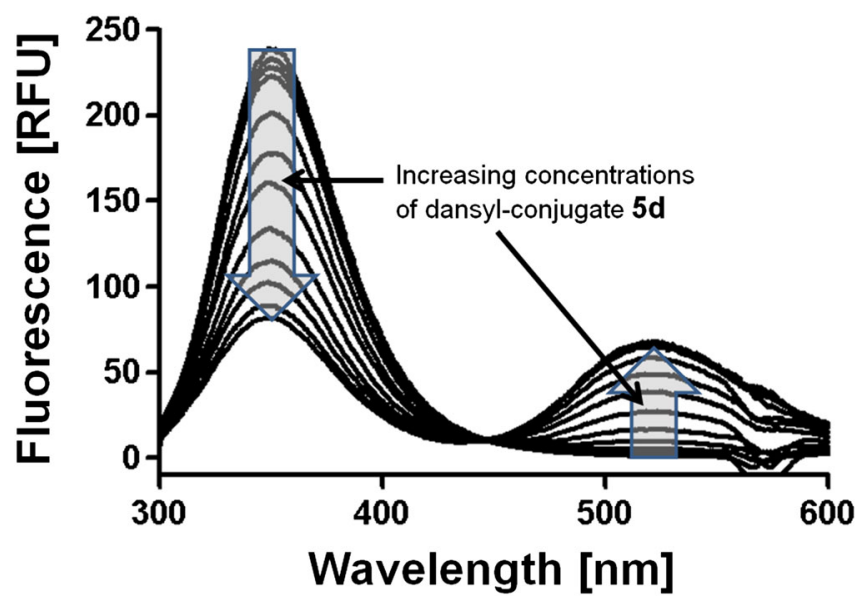

Figure 2. Fluorescence resonance energy transfer between $\mathrm{HDAH}$ and 5d. Titration of $100 \mathrm{nM}$ HDAH in assay buffer with increasing concentrations of $\mathbf{5 d}$ ranging from $12.5 \mathrm{nM}$ to $12.8 \mu \mathrm{M}$ at $25^{\circ} \mathrm{C}$. The samples were excited at $285 \mathrm{~nm}$, and the emission spectra corrected for contributions of direct excitation of the ligand. The fluorescence emission intensity was plotted versus wavelength. The signal at about $350 \mathrm{~nm}$ decreased, and the emission at about $525 \mathrm{~nm}$ increased upon binding of $\mathbf{5 d}$ to $\mathrm{HDAH}$, which was indicative for fluorescence resonance energy transfer. The perturbation at about $570 \mathrm{~nm}$ was due to a suboptimal correction of second order diffraction at the excitation grating monochromator.

bound to HDAH with a dissociation binding constant, $K_{d}$, above $20 \mu \mathrm{M}$, whereas binding of $\mathbf{5 a}$ was not detectable up to concentrations of $50 \mu \mathrm{M}$. The weak interactions observed for ligands $\mathbf{5 a}$ and $\mathbf{5 b}$ were probably caused by the short spacer length of $n=2$ and 3 between the metal ion chelating hydroxamate group and the bulky dansyl residue, which hampered the access of the ligand to the bottom of the binding pocket due to sterical hindrance thereby interfering with the key electrostatic interaction between the zinc cation at the base of the active site pocket and the zinc-binding hydroxamate functionality of the ligand. Dansyl-conjugates $\mathbf{5 c - 5 f}$ bound to HDAH with increasing affinity down to a $K_{d}$ value of $60 \pm 7 \mathrm{nM}$ for $\mathbf{5 f}$ at $25^{\circ} \mathrm{C}$ (Figure 3). Many HDAC inhibitors have been reported that consist of a hydroxamic acid and a five-carbon or six-carbon hydrophobic linear spacer attached to an aromatic surface recognition motif

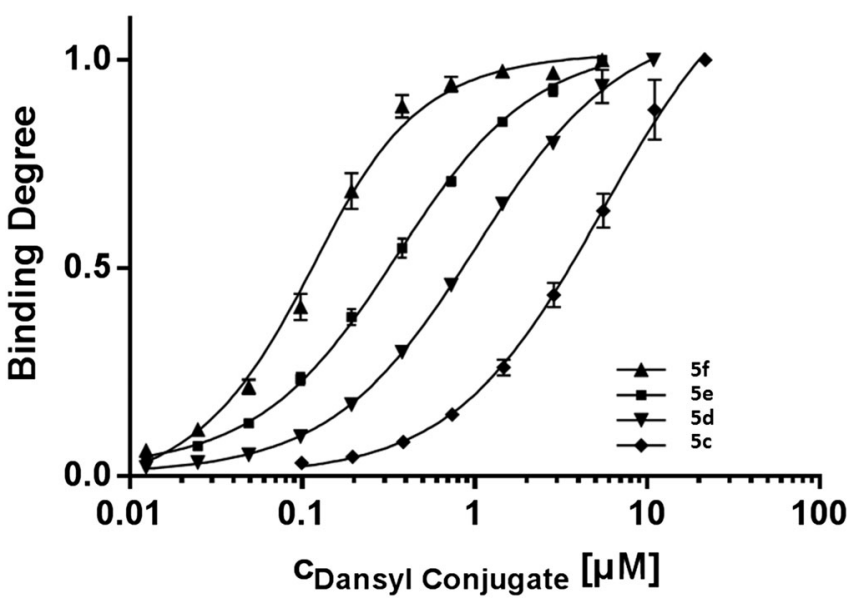

Figure 3. Binding isotherms of dansyl ligands $\mathbf{5} \mathbf{c}-\mathbf{f}$ to HDAH. Binding degrees were plotted versus the respective ligand concentration as indicated. Indicated concentrations of $\mathbf{5 c}-\mathbf{f}$ were added to $100 \mathrm{nM} \mathrm{HDAH}$ in assay buffer at $25^{\circ} \mathrm{C}$. The binding degree was calculated from the change in FRET signal upon binding as described in the method section. All data points represent means of triplicates. The standard errors are displayed as error bars. The data were fit to a one-site binding model considering ligand depletion (equation 3 ) as described in the experimental section. The calculated $K_{d}$ values are summarized in Table 1.

(Paris et al., 2008). This general design criterion is confirmed by our series of dansyl-ligands with affinity increasing for linkers extending from two to seven-carbon units. Binding of $\mathbf{5 c - 5 f}$ to $\mathrm{HDAH}$ was reversible as shown by the displacement of the dansyl-ligands with SAHA, which effectively competed for binding in the active site of the enzyme. Such competitive binding allowed the use of these fluorescent ligands in further competitive binding experiments with known inhibitory compounds. The drug SAHA and the known HDACi MB275 were chosen as test compounds. We previously reported the synthesis and HDAC inhibiting features of MB275, which is a hybrid compound of the natural product HDAC inhibitor psammaplin A (Baud et al., 2012). Binding constants of both non-fluorescent inhibitors were determined from competitive titration experiments where the compound was titrated into a pre-formed complex consisting of dansyl-ligand probe $\mathbf{5 e}$ and HDAH (Figure 4). We determined 


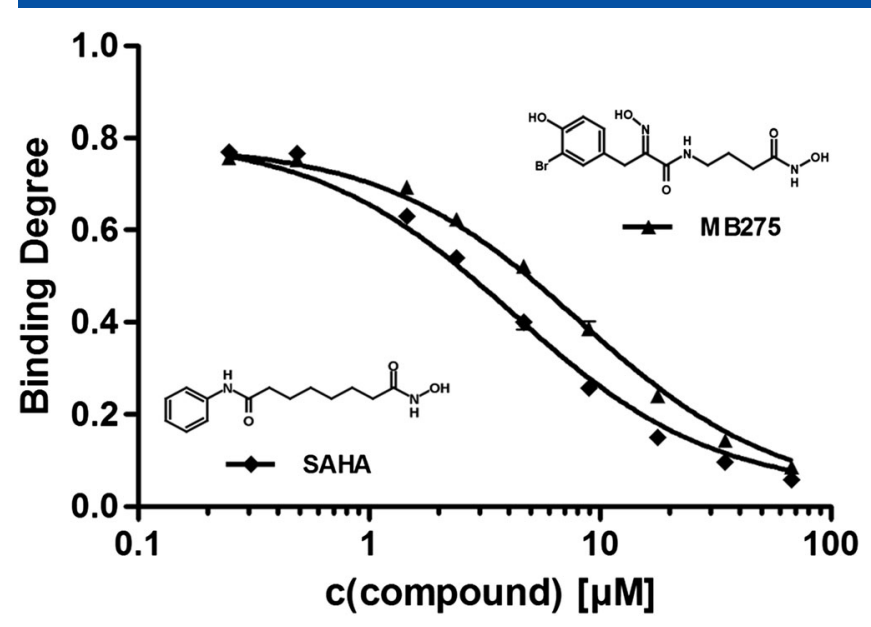

Figure 4. Competitive displacement of $\mathbf{5 e}$ from HDAH. Binding degrees calculated from FRET signals were plotted against the concentration of non-fluorescent HDAH inhibitors. 1 mikromolar $(\mu \mathrm{M}) \mathbf{5 e}$ and $100 \mathrm{nM} \mathrm{HDAH}$ were mixed to pre-form about $80 \%$ of its complex with HDAH in assay buffer. Subsequently, different concentrations of SAHA and MB275 were added. Each data point represents the mean of triplicates. Error bars indicate the standard error. In most cases, the error becomes so small that error bars are indistinguishable from corresponding data points. The data were fitted to a competitive binding model (equation 4) as described in the experimental section. The $K_{d}$ values of SAHA and MB275 were calculated to be $0.96 \pm 0.01$ and $2.1 \pm 0.2 \mu \mathrm{M}$, respectively.

exemplarily $K_{d}$ values of both inhibitors at eight different temperatures to demonstrate that thermodynamic parameters could also be obtained from the temperature dependency of

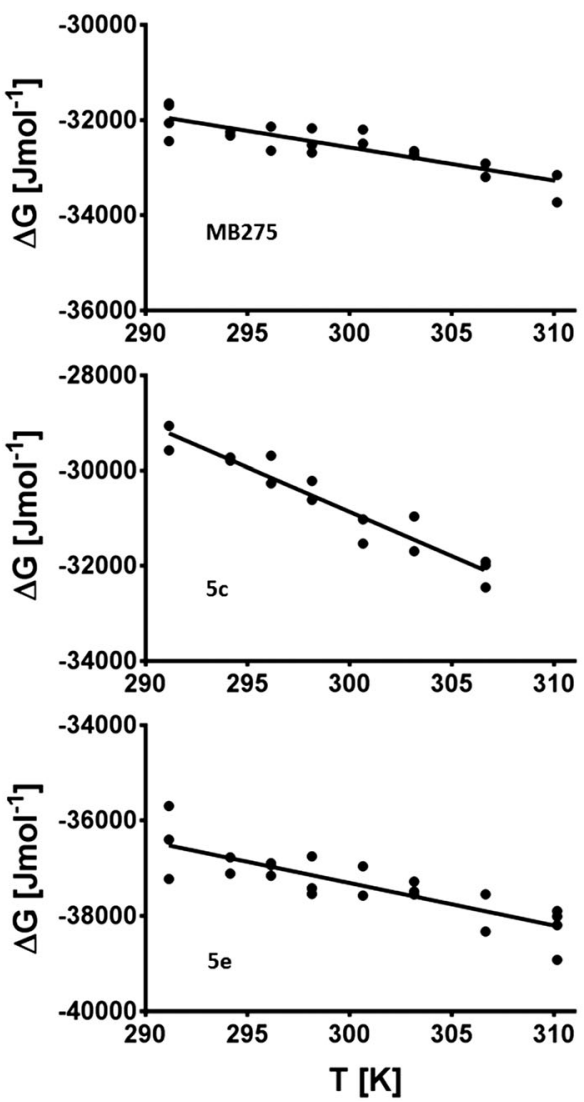

$K_{d}$ as calculated from indirect competitive binding assays. The binding constant obtained for SAHA, $K_{d}=0.96 \pm 0.01 \mu \mathrm{M}$, is in good agreement with $1.0 \mu \mathrm{M}$ as determined by a recently reported FRET-assay exploiting a furylacryloyl group as an acceptor chromophor that does not emit fluorescence (Riester et al., 2007) and $0.77 \mu \mathrm{M}$, which was obtained from a combined fluorescence polarization and lifetime-based binding assay (Riester et al., 2009).

The robustness and performance of the FRET-assay in highthroughput applications were also demonstrated using microtiter plates (Figure S6). The performance of the high throughput screening (HTS) assay was calculated according to Zhang et al. (1999). For the FRET-based binding assay, excellent performance was proven by $z^{\prime}$-values between 0.72 and 0.82 . We achieved a manual throughput of 96 compounds in about $50 \mathrm{~min}$. The limiting step was a 30-min incubation of the preformed protein-ligand complex and the screening compounds to reach chemical equilibrium. Using specialized robotic equipment and more dense plate formats a much higher throughput will be possible.

\section{Thermodynamic signatures and selectivity of histone deacetylase like amidohydrolase ligands}

Van't Hoff plots of dansyl-conjugate ligands $\mathbf{5 c - f}$ and inhibitors SAHA and MB275 are shown in Figure 5. All data showed a linear relationship between $\Delta \mathrm{G}^{0}$ and $\mathrm{T}$ and negative slope indicating an increase in entropy upon binding of ligands to HDAH. The enthalpic and entropic contributions are presented in Figure 6. The relatively weak binding of $\mathbf{5 c}$ to $\mathrm{HDAH}$ is clearly entropically driven as indicated by a distinct positive binding enthalpy.

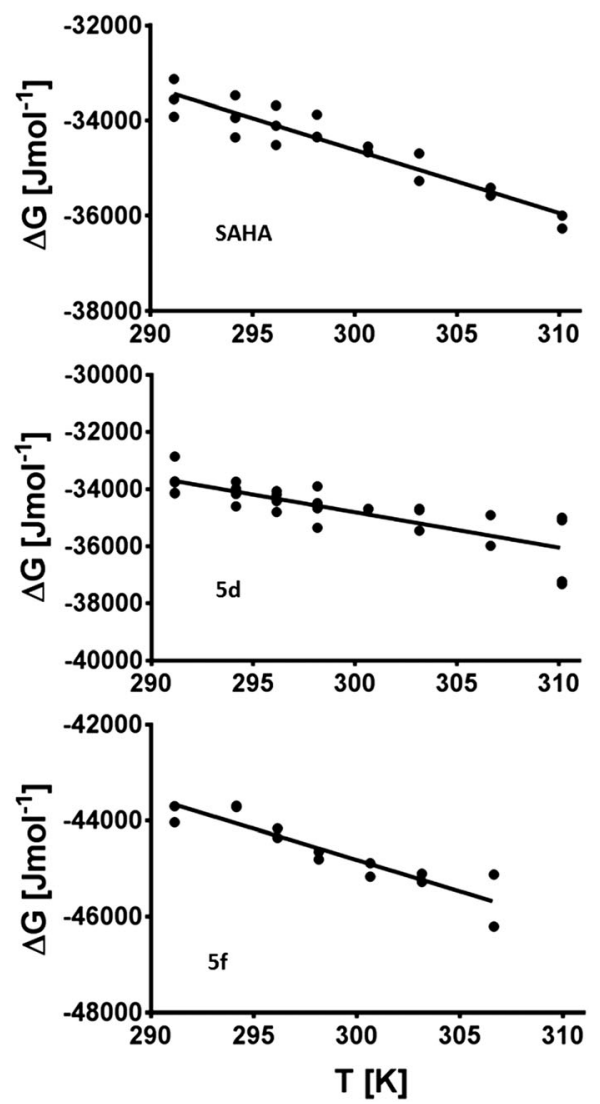

Figure 5. Van't Hoff plots of ligand binding to HDAH. Free binding energy, $\Delta G^{0}$, derived from equilibrium titrations involving indicated compounds were plotted versus temperature. The data were fitted to the expression in equation 2 yielding $\Delta \mathrm{H}^{0}$ and $\Delta S^{0}$. 


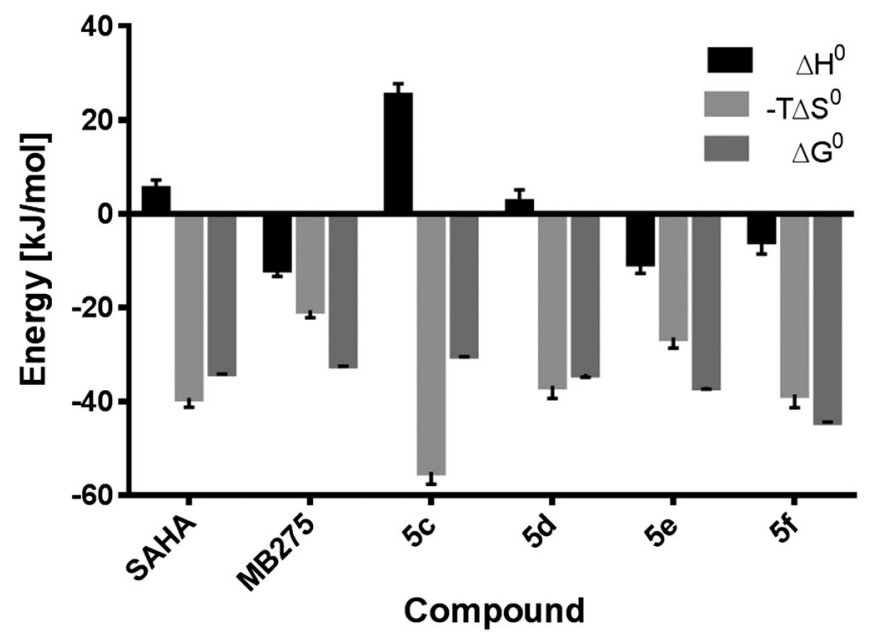

Figure 6. Thermodynamic signatures of HDAH ligands at $295 \mathrm{~K}$.

Increasing the length of the linker yields not only stronger binding but also a shift to enhanced enthalpic contribution to the recognition of $\mathrm{HDAH}$ and ligand. On the other hand, the binding affinity and the $\Delta H^{0} / \Delta G^{0}$ ratio are not perfectly correlated. Even though $\mathbf{5 f}$ showed a 5 -time lower $K_{d}$-value than $\mathbf{5 e}$, 5 e turned out to feature the highest enthalpy contribution to the free energy of binding. Interestingly, the binding of the approved drug SAHA to HDAH was entropically driven with a small but significant unfavorable positive binding enthalpy. In contrast, the derivative of psammaplin A, MB275, showed a similar affinity but a much higher enthalpic contribution to binding. The importance of favorable $\Delta \mathrm{H}^{0}$ is highlighted by several authors (Freire, 2008; Ladbury et al., 2009). It is postulated that favorable enthalpic contributions reflect optimal geometries in noncovalent interactions particularly hydrogen-bonds and $\mathrm{vdW}$ interactions. The optimal molecular recognition is achieved, if all polar atoms of the binding site are satisfied with polar partners of the ligand having all $\mathrm{H}$-Bonds formed perfectly directed at optimal distances and vdW forces are maximized by a perfect geometric fit between protein and ligand. Favorable entropic terms arise mainly from desolvation effects or a net increase in mobility of the binding partners. Maximal selectivity can be achieved, if few strong $\mathrm{H}$-bonds with defined angles and interatomar distances, which are indicated by a favorable enthalpic contribution, toward unique sites in a target protein are formed, which are not possible in off-target molecules. On the other hand, the major entropic contribution to binding affinity often results from the hydrophobic effect, which is associated with positive binding entropy and represents the exclusion of solvent molecules rather than a directed attractive force between protein and ligand. Therefore, entropic contributions to binding affinity are usually non-selective in nature. In addition, conformationally constrained inhibitors with perfect shape complementarity to a binding pocket will have better binding affinities and also selectivities than their flexible derivatives because of a reduced loss of conformational entropy and therefore a greater enthalpic contribution to binding. While the optimization of $\Delta \mathrm{H}^{0}$ does not necessarily increase ligand affinity due to enthalpy-entropy compensation, it is supposed to increase selectivity due to the spatial and electrostatic requirements at the protein-ligand interaction surface (Chaires, 2008; Kawasaki and Freire, 2011). In addition, Ohtaka and Freire observed that HIV-protease inhibitors, which established strong enthalpic ( $\mathrm{H}$-bonds and vdW) interactions with conserved regions of the target protein and contained flexible functionalities, achieved high adaptability to mutant proteins while maintaining selectivity against off-target proteins (Ohtaka and Freire, 2005). To analyze the relationship between enthalpic contribution and selectivity, the capability of all ligands to inhibit representatives of human HDAC classes I and II was investigated using enzyme activity assays as described in the method section. The resulting IC50-values are summarized in Table 1. The series of dansylligands that are characterized by the same $\mathrm{Zn}^{2+}$-chelating hydroxamate and dansyl-head group and only differ in the length of the alkyl linker was analyzed in more detail. Ratios of IC50-values served as a measure for selectivity in terms of enzyme inhibition and were correlated with the enthalpic contribution to free energy of binding, $\Delta H^{0} / \Delta G^{0}$ (Figure 7). HDAH is a close homologue of HDAC6 and therefore belongs to HDAC class Ilb. The data in Figure 7 highlight the clear correlation between selectivity against HDAC1 as a representative of HDAC class I and the $\Delta H^{0} / \Delta G^{0}$ ratio. 5e achieves a ratio of 0.29 , which is in agreement with Ruben et al. (2006), who reported that the optimal enthalpic contribution to binding is about one-third for the interaction between allophenylnorstatine inhibitors and plasmepsin II. However, the optimal enthalpy-entropy balance in molecular recognition between protein and ligands is supposed to be dependent on the chemistry and geometry of the binding site. In contrast to the increase in selectivity against

Table 1. Binding constants and IC50 values against histone deacetylase like amidohydrolase and histone deacetylase enzymes

\begin{tabular}{|c|c|c|c|c|c|c|c|}
\hline Ligand & $n$ & $\mathrm{IC} 50 / \mu \mathrm{M}$ HDAH & $\mathrm{IC} 50 / \mu \mathrm{M}$ HDAC1 & IC50/ $\mu \mathrm{MHDAC} 4$ & 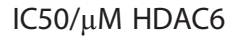 & $\mathrm{IC} 50 / \mu \mathrm{M}$ HDAC8 & $\mathrm{K}_{\mathrm{d}} / \mu \mathrm{M} \mathrm{HDAH}$ \\
\hline $5 a$ & 2 & $7.8 \pm 0.2$ & $>50$ & $>100$ & $>100$ & $6.7 \pm 1.9$ & $>50$ \\
\hline $5 b$ & 3 & $0.97 \pm 0.18$ & $2.0 \pm 0.2$ & $86 \pm 24$ & $3.1 \pm 0.3$ & $2.4 \pm 0.8$ & $>20$ \\
\hline $5 c$ & 4 & $0.30 \pm 0.02$ & $0.18 \pm 0.05$ & $59 \pm 7$ & $0.41 \pm 0.04$ & $1.8 \pm 0.3$ & $5.4 \pm 0.7$ \\
\hline $5 d$ & 5 & $0.08 \pm 0.01$ & $0.13 \pm 0.02$ & $28 \pm 2$ & $0.39 \pm 0.04$ & $0.68 \pm 0.10$ & $0.96 \pm 0.05$ \\
\hline $5 e$ & 6 & $0.060 \pm 0.002$ & $0.19 \pm 0.04$ & $10 \pm 1$ & $0.22 \pm 0.05$ & $0.32 \pm 0.04$ & $0.30 \pm 0.02$ \\
\hline $5 f$ & 7 & $0.056 \pm 0.002$ & $0.17 \pm 0.02$ & $5.0 \pm 0.2$ & $0.080 \pm 0.010$ & $0.23 \pm 0.02$ & $0.060 \pm 0.007$ \\
\hline SAHA & - & $0.063 \pm 0.002$ & $0.017 \pm 0.001$ & $>50$ & $0.046 \pm 0.002$ & $1.4 \pm 0.1$ & $0.96 \pm 0.01$ \\
\hline MB275 & - & $0.060 \pm 0.001$ & $0.34 \pm 0.01$ & $>100$ & $2.4 \pm 0.1$ & $9.3 \pm 0.5$ & $2.1 \pm 0.2$ \\
\hline
\end{tabular}

Compounds were tested in enzyme activity assays under conditions described in the method section. Mean values and standard errors were calculated from fits to sets of 10 different concentrations for each ligand and compound.

$\mathrm{HDAH}$, histone deacetylase like amidohydrolase; HDAC, histone deacetylase; SAHA, suberoylanilide hydroxamic acid. 

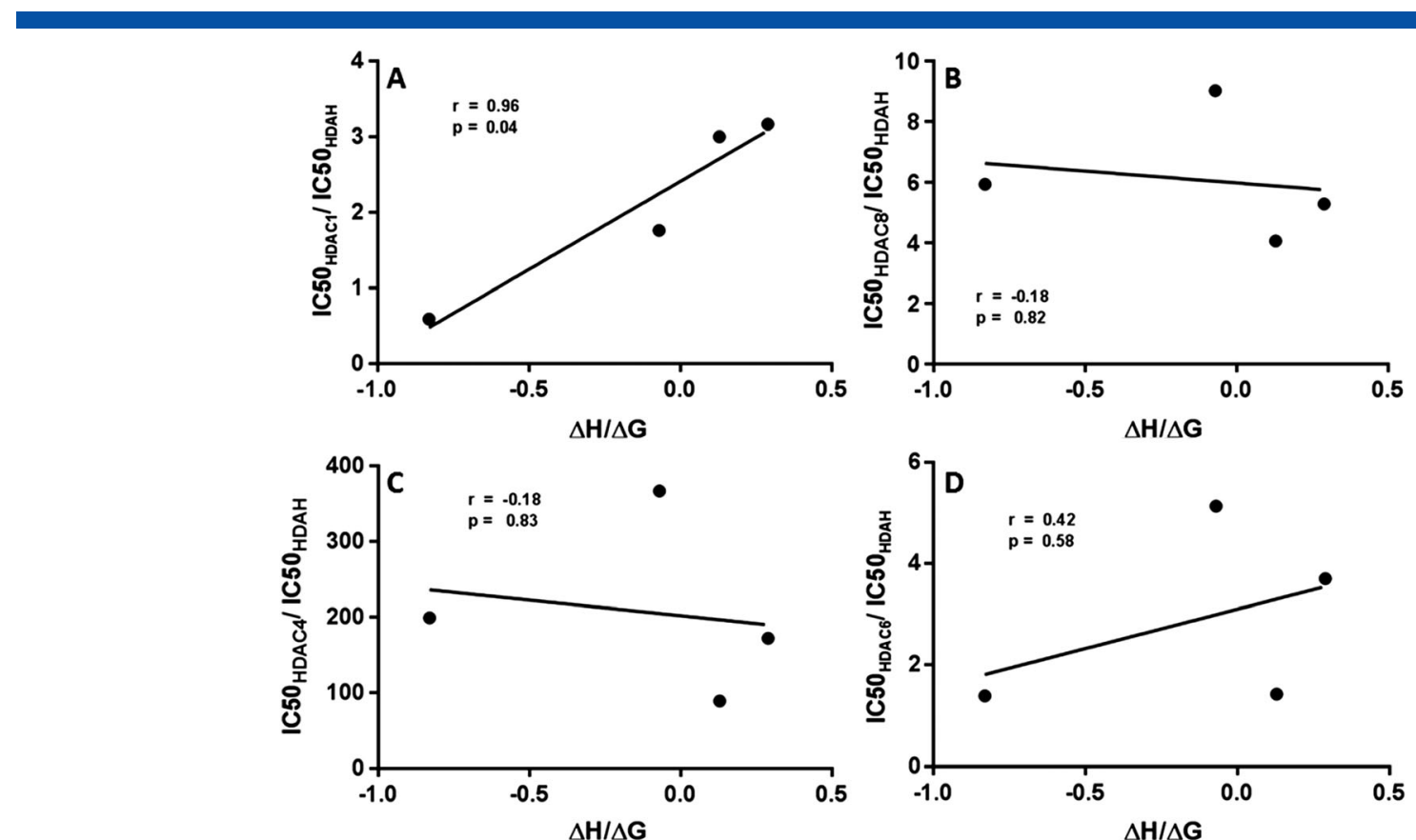

Figure 7. Relationship between selective inhibition and thermodynamic signatures. The ratio of IC50-values against HDAH and different HDACs is considered as a measure of selective enzyme inhibition and plotted versus the ratio $\Delta \mathrm{H}^{0} / \Delta \mathrm{G}^{0}$. The Pearson coefficient $r$ indicates the correlation between selectivity versus the respective human HDAC and the enthalpic contribution to binding. The relationship is significant, if the $p$-values $<0.05$.

HDAC1, the selectivity against the closest human homologue, HDAC6, could not be improved. This finding underlines the sequential and structural similarities within the same HDAC class, which does not provide a good starting point for selectivity improvements between class members. Furthermore, there is only a slight but not significant increase in selectivity against HDAC8 with higher contribution of $\Delta \mathrm{H}$ to binding. This could be explained by the fact that HDAC8 is the most distant member of class I HDACs and also shows features of class II HDACs. Among others, the substrate Boc-Lys(trifluoacetyl)-AMC is converted by class II enzyme HDAC4 and HDAC8, but not by $\mathrm{HDAC1}$; on the other hand, the unfluorinated derivative is an excellent substrate of HDAC1 but not of HDAC4 and HDAC8. The ambivalent functional characteristics and structural features of HDAC8 make it more difficult to achieve selectivity improvements regarding the inhibition of HDAH and HDAC8. There is already a big selectivity difference between the inhibition of HDAC4 and all other enzymes. This is attributed to the unique substitution of the tyrosine (Y312 in HDAH) in the catalytic center by histidine in human class Ila HDACs, which causes a dramatic loss in affinity for many hydroxamate-based inhibitors, including the rather unselective so-called pan-inhibitor SAHA and MB275. In the light of the distinct molecular differences of the binding pockets and the ensuing tremendous selectivity of all investigated ligands against HDAC4, no further improvement could be achieved. Altogether, the data are consistent with the classification of HDACs and underline the suitability of thermodynamic signatures as guidance for the optimization of the selectivity of inhibitors.

\section{Molecular dynamics simulations}

Because proteins are flexible and supposed to undergo more or less pronounced conformational changes and induced fit upon ligand binding, docking and MD simulations were performed to obtain a dynamic view on the HDAH-ligand complexes and the involved distinct intermolecular interactions. The computational analysis of the MD simulations allowed the interpretation of the thermodynamic data in terms of the structural and dynamic information on the complexes. The electrostatic interaction between the hydroxamate group of the ligands and the catalytic $\mathrm{Zn}^{2+}$ ion, hydrogen bonds to mainly Y312 and H142 as well as vdW interactions are the main determinants of recognition that govern the binding enthalpy of ligand binding to $\mathrm{HDAH}$. For a molecular interpretation of the experimental thermodynamic parameters, the X-ray structure of the complex between HDAH and SAHA (PDB 1ZZ1) was used to dock all investigated ligands to HDAH. The complexes were energetically minimized and subjected to MD simulations in water environment with adapted $\mathrm{NaCl}$ concentration to analyze the dynamics of the elementary molecular interactions in more detail. Besides fluctuation information and evidence for structural water, the analysis revealed the number of ligand- $\mathrm{Zn}^{2+}$ contacts, percentages of $\mathrm{H}$-bonds, and the mean number of vdW contacts over the whole time range of simulations (Table 2). The resulting data suggest that the dansyl-ligands $\mathbf{5 d - 5 f}$ as well as SAHA and MB275 form bi-dentate complexes with the $\mathrm{Zn}^{2+}$ ion at the bottom of the active site thereby effectively shielding the metal ion from solvent water molecules (Figure $8 \mathrm{~A}$ and $8 \mathrm{C}$ ). All ligands, even the weak micromolar binders, tighten the binding pocket significantly causing entropic losses. The dansyl ligands with highest affinity to $\mathrm{HDAH}, \mathbf{5} \mathbf{f}$ and $\mathbf{5 e}$, displayed strong favorable changes in binding enthalpy, $-5.7 \pm 2.9$ and $-10.5 \pm 2.3 \mathrm{~kJ} \mathrm{~mol}^{-1}$, respectively. This correlated with the highest mean number of vdW contacts, two contacts to $\mathrm{Zn}^{2+}$, and high percentage of $\mathrm{H}$-bonds to $\mathrm{Y} 312$ or H142. 5e showed a distinct low residual mobility and also reduced the RMSF of the flexible loop (90-104) strongest which agrees with the lowest favorable binding entropy. Compared with $\mathbf{5 e}$, the residual flexibility of the ligand and loop (90-104) in the complex between $\mathbf{5 f}$ and HDAH was increased, 


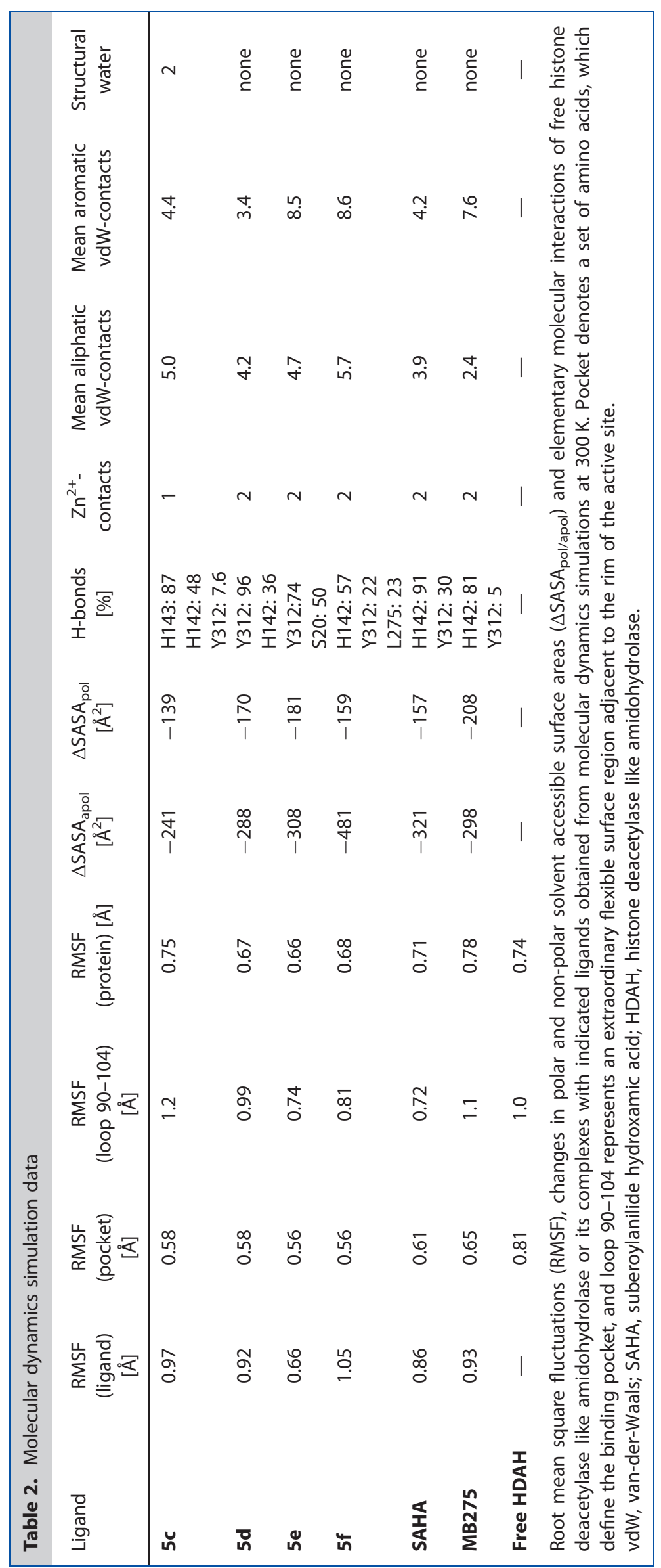




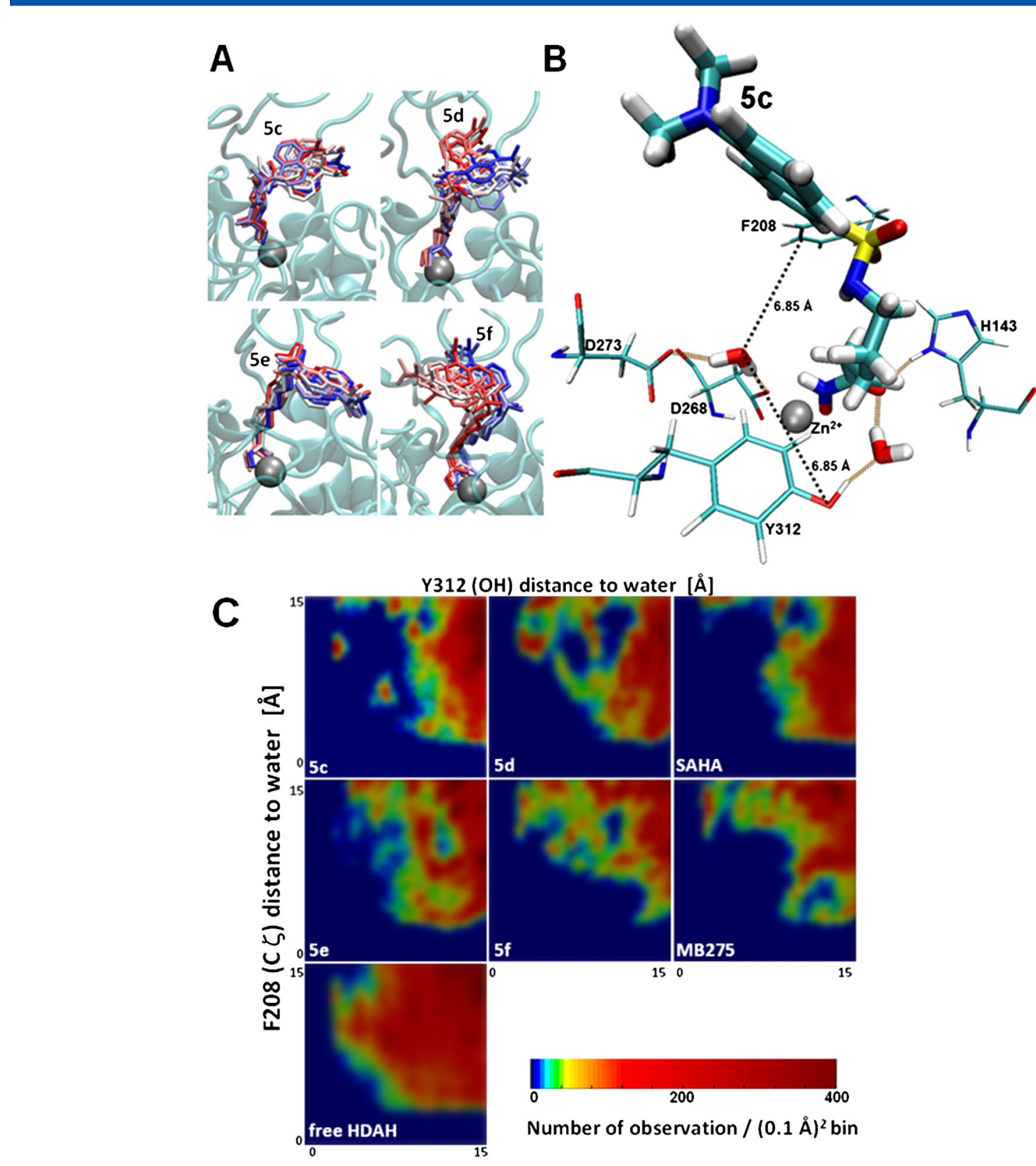

Figure 8. Molecular dynamics simulation of dansyl-ligand complexes with $\mathrm{HDAH}$. A, overlay of a series of snapshots of dansyl-ligands $\mathbf{5 c - f}$ in the active site of HDAH. The different colors of the corresponding ligands indicate snapshots at different simulation times (11-20 ns in $1 \mathrm{~ns}$ steps) and illustrate the flexibility of the ligand within the binding pocket; B, structural water molecules within the binding pocket of the complex between $\mathbf{5 c}$ and HDAH. Purple lines between water molecules and protein or ligand represent hydrogen bonds. C, water distribution within the binding pocket of HDAH, unbound or in complex with indicated ligands. Data are from 2000 snap shots of MD simulation for each system.

which is attributed to the long spacer and enhanced fluctuation of the only weakly constrained dansyl head group. Consequently, the entropic penalty was significantly reduced, while the enthalpic contribution was only slightly decreased yielding a higher affinity than $\mathbf{5 e}$. Dansyl-ligand $\mathbf{5 d}$ showed a slightly positive binding enthalpy, which is compensated by a favorable binding entropy indicating a loss of strong specific interactions. The positive $\Delta \mathrm{H}^{0}$ corresponds mainly to a decrease in vdW contacts to the aromatic side chains of F208 and F152. The loss in vdW contacts accounts for an increased remaining mobility of the ligand, which is supposed to increase the corresponding binding entropy. In addition, binding of $\mathbf{5} \mathbf{d}$ did not tighten the flexible loop (90-104) thereby further raising $\Delta S^{0}$ with respect to $\mathbf{5 e}$ and $\mathbf{5 f}$. The clearly decreased affinity of $\mathbf{5 c}$ is characterized by a largely unfavorable binding enthalpy and a large compensating positive binding entropy. The dramatic enthalpic loss agrees with the ability of $\mathbf{5 c}$ to form only a monodentate complex with $\mathrm{Zn}^{2+}$, which is accompanied by a further loss of hydrogen-bonding to $\mathrm{Y} 312$, which is a very important determinant of inhibitor binding to HDAH in X-Ray structures (PDB 1ZZ1, $1 Z Z 3$, and $2 \mathrm{VCG})$. This weak coordination is relevant to the limited water access to the binding pocket. In Figure $8 \mathrm{C}$, the water distribution within the binding pocket of free HDAH and all investigated ligand-protein complexes is shown. The distribution maps represent the density of water molecules at positions with indicated distances to the oxygen atom of the hydroxyl group of Y312 at the bottom and the aromatic $C \zeta$ atom of F208 at the rim of the 
active site. The distribution patterns reveal that water molecules are largely displaced upon ligand binding, as expected. On the other hand, different ligands cause differential water distribution patterns, which could be attributed partially to the space-filling properties of the ligands but also to the adaptation of the binding pocket to the respective ligand. It is also striking to see two distinct small well-defined areas of high solvent density within the binding pocket of dansyl-ligand $\mathbf{5 c}$, which cannot be seen with all other ligands (Figure $8 \mathrm{C}$ ). Similar but less sharply contoured areas of higher solvent density near $\mathrm{Y} 312(\mathrm{OH})$ in the binding pockets of 5d or MB275 represent water molecules that only occupy this space for short timescales, being long-term exchanged for alternative water molecules. The two spots with increased solvent density in the solvent distribution map of the $\mathbf{5} \mathbf{c} / \mathrm{HDAH}$ complex represent two water molecules that are trapped at certain positions within the binding pocket and can effectively be regarded as structural water molecules held in place by hydrogen-bonding throughout a time range of 40 ns MD simulation at $300 \mathrm{~K}$ (Figure $8 \mathrm{~B}$ and Figure S3). The first structural water molecule bridges HDAH and ligand through hydrogen-bonds between the hydroxyl-oxygen of Y312 and the carbonyl-oxygen of $\mathbf{5 c}$ thereby stabilizing the mono-dentate electrostatic interaction between $\mathbf{5 c}$ and $\mathrm{Zn}^{2+}$. The water molecule has a mean distance of $2.8 \AA$ to the hydroxyl-oxygen $(\mathrm{OH})$ of $\mathrm{Y} 312$ and $11 \AA$ to the $C \zeta$ atom of F208. The second spatially constrained water molecule is hydrogen-bonded by D273 and D268 with a mean distance of $6.9 \AA$ to both $\mathrm{Y} 312(\mathrm{OH})$ and $\mathrm{F} 208(\mathrm{C \zeta})$. The deficit in specific interactions correlates with overall increased RMSF values indicating higher flexibility. The affinity of SAHA and MB275 is rather similar to dansyl-ligand $\mathbf{5 d}$ as is the chelation of $\mathrm{Zn}^{2+}$ and the mean number of vdW-contacts. At the same time, binding to HDAH appears more entropy driven for $\mathbf{5 d}$ and SAHA than for MB275 at $25^{\circ} \mathrm{C}$. These disparities are hard to interpret because of larger differences in the chemical structures of the respective ligands, which differ more significantly than only in the length of the spacer. Hydrophobic interaction of the dansyl-ring of $\mathbf{5 d}$ with hydrophobic amino acid side chains and release of solvated water molecules is a potential source for a more positive $\Delta S^{0}$.

In general, the enthalpy/entropy balance is understood to depend on many system-specific properties (Bissantz et al., 2010) but is still accepted as an important factor for optimizing the selectivity of drugs and avoiding side effects (Kawasaki and Freire, 2011) thus aiding the decision about which compound to take forward in the drug development process. If several compounds are available with similar binding constants but different thermodynamic signatures, as is the case with $\mathbf{5 d}$, SAHA, and MB275, a greater enthalpic contribution to binding is supposed to be more beneficial in terms of selective recognition.

\section{Association and dissociation kinetics of dansyl-ligands and histone deacetylase like amidohydrolase}

It is not only of interest to analyze a ligand-protein system at chemical equilibrium but rather to also investigate its kinetic behavior. The investigation of association and dissociation kinetics provides more detailed information about catalytic intermediates and reaction mechanisms. Moreover, the determination of rate constants of elementary compoundtarget protein reaction steps will provide valuable additional information for the assessment of potential drug candidates. In this study, we demonstrate the general applicability of the FRET-based binding assay to the measurement of binding kinetics. For dansyl-ligands $\mathbf{5 c - f}$, both association and dissociation kinetics could be measured (Figure 9 Figure S2). The dissociation rate constants of $\mathbf{5 c}, \mathbf{5 d}, \mathbf{5 e}$, and $\mathbf{5 f}$ decreased with increasing spacer length and were determined to be $(15.6 \pm 0.1) \times 10^{-3} \mathrm{~s}^{-1}, \quad(5.0 \pm 0.02) \times 10^{-3} \mathrm{~s}^{-1}$, $(4.0 \pm 0.01) \times 10^{-3} \mathrm{~s}^{-1}$, and $(0.9 \pm 0.004) \times 10^{-3} \mathrm{~s}^{-1}$ at $21^{\circ} \mathrm{C}$, respectively. Further studies with varying concentrations of these dansyl-ligands are ongoing to elucidate the respective binding mechanism to HDAH in more detail.

Usually, the kinetics of binding reactions are investigated by mixing both reaction partners and measuring the change of an optical signal indicating the progression of complex formation as was demonstrated for aforementioned dansyl-ligands and HDAH. However, it would be even more interesting to investigate the binding kinetics of potential drugs to HDAH, if no optical change could be observed upon complex formation. In principle, a variant of the described homogeneous FRET-based binding assay should also be applicable to the indirect dissection of such drug-HDAH binding kinetics through the observation of the concurrent displacement of the dansyl-ligand probe. An
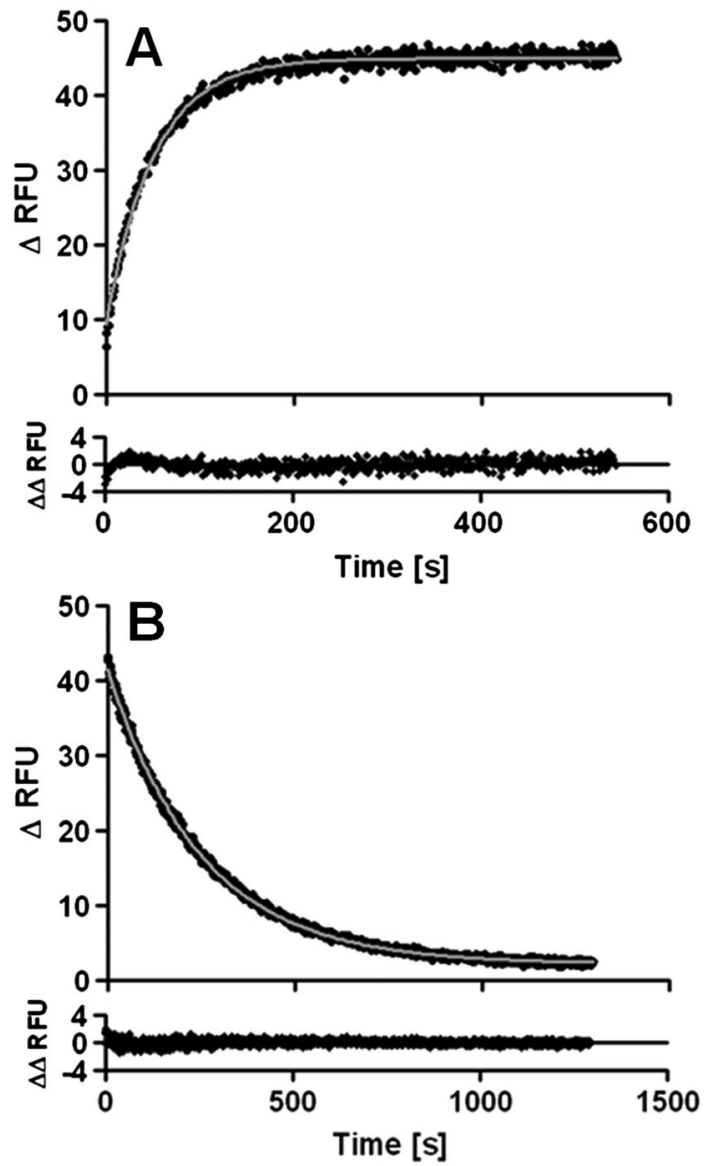

Figure 9. Association and dissociation kinetics of $\mathbf{5 e}$ binding to $\mathrm{HDAH}$. A, association kinetics of $200 \mathrm{nM} \mathrm{HDAH}$ and $1.6 \mu \mathrm{M} \mathbf{5 e}$ in assay buffer. $B$, dissociation kinetics of pre-formed complex in the reaction of panel A initiated by the addition of $500 \mu \mathrm{M}$ SAHA. The first $4 s$ of the kinetics were missing because of mixing artifacts. All reactions were performed in assay buffer at $21^{\circ} \mathrm{C}$. Fluorescence was excited at $285 \mathrm{~nm}$, and the change in fluorescence intensity at $525 \mathrm{~nm}(\Delta \mathrm{RFU})$ was plotted versus time. The smooth dark gray lines represent non-linear regression fits to mono-exponential functions. 
important prerequisite for such an experimental setup is that the dissociation of the ligand-probe, in our case the dansyl-ligand, from its complex with HDAH is not the rate limiting step (see, e.g., Neumann et al., 2011). A glance at the dissociation rates of the dansyl-ligand probes revealed that $\mathbf{5 c}$ showed the fastest dissociation behavior and could be used to measure association and dissociation rate constants of any non-fluorescent compound at conditions where its binding to HDAH occurs much slower than the dissociation of the probe.

\section{CONCLUSIONS}

A homogeneous competitive FRET-based binding assay was developed to determine thermodynamic parameters for the binding of dansyl-ligands and non-fluorescent compounds of interest like SAHA to HDAH, using the van't Hoff approach. In contrast to micro-calorimetric studies, the presented assay format enables a high-throughput analysis of larger numbers of hit compounds from pharmaceutical screening campaigns on microtiter plates with extremely low consumption of target protein, ligand-probe, and compound. The fast readout of the assay can also be used for further detailed mechanistic studies of binding kinetics, even on millisecond time scales. Moreover, the assay principle should be transferable to human recombinant HDACs, which are known to be inhibited by SAHA, allowing the determination of thermodynamic signatures for inhibitors of this important class of cancer targets. X-ray structures of $\mathrm{HDAH}$ complexes with several hydroxamate ligands as well as docking and MD simulations suggest a bi-dentate coulombic interaction between the dansyl-ligand probes and the $\mathrm{Zn}^{2+}$ ion at the bottom of the active site, if the ligands contain alkyl spacer with more than four carbon atoms. Dansyl-ligand $\mathbf{5 c}$ with a 4-carbon alkyl-spacer was only able to form a mono-dentate complex with the $\mathrm{Zn}^{2+}$ ion. Although the affinity increased for dansyl-ligands with increasing length of the alkyl spacer, the maximal enthalpic contribution to the free energy of binding of $29 \%$ was observed with the dansyl-ligand containing a hexyl spacer. The binding data of dansyl-ligands to HDAH clearly demonstrated a positive correlation between the $\Delta H^{0} / \Delta G^{0}$ ratio and selectivity against HDAC1 as representative of class I HDACs, whereas no improved selectivity was observed against enzymes out of the same HDAC class as HDAH. This observation suggests that thermodynamic signatures can be used to guide the optimization of selectivity within a series of structurally related inhibitors. Therefore, we are convinced that thermodynamic signatures are supposed to provide additional particularly meaningful parameters for the selection of drug candidates with presumably reduced risk to fail in subsequent more expensive clinical phases.

\section{Acknowledgements}

The work has been supported by a grant of the Landesoffensive zur Entwicklung Wissenschaftlich-oekonomischer Exzellenz (LOEWE Soft Control) to Franz-Josef Meyer-Almes and by the Association for International Cancer Research (AICR grant 08-0407) to Matthew J. Fuchter. The excellent support of Michael Schröder is greatfully acknowledged.

\section{REFERENCES}

Aldana-Masangkay Gl, Sakamoto KM. 2011. The role of HDAC6 in cancer. J. Biomed. Biotechnol. 2011: 1-10. DOI: 10.1155/2011/875824.

Baud M, Leiser T, Haus P, Samlal S, Wong AC, Wood R, Petrucci V, Gunaratnam M, Hughes S, Buluwela L, Turlais F, Neidle S, MeyerAlmes FJ, White AJ, Fuchter MJ. 2012. Defining the Mechanism of Action and Enzymatic Selectivity of Psammaplin A Against its Epigenetic Targets. J. Med. Chem. 55: 1731-1750. DOI: 10.1021/ jm2016182.

Bissantz C, Kuhn B, Stahl M. 2010. A medicinal chemist's guide to molecular interactions. J. Med. Chem. 53: 5061-5084. DOI: 10.1021/jm100112j.

Bronowska AK. 2011. Thermodynamics of ligand-protein interactions: implications for molecular design. InTech 1-48. DOI: 10.5772/19447.

Chaires JB. 2008. Calorimetry and thermodynamics in drug design. Annu. Rev. Biophys. 37: 135-151. DOI: 10.1146/annurev. biophys.36.040306.132812.

DePaul AJ, Thompson EJ, Patel SS, Haldeman K, Sorin EJ. 2010. Equilibrium conformational dynamics in an RNA tetraloop from massively parallel molecular dynamics. Nucl. Acids Res. 38: 4856-4867. DOI: $10.1093 /$ nar/gkq134.

Freire E. 2008. Do enthalpy and entropy distinguish first in class from best in class? Drug Discov. Today 13: 869-874. DOI: 10.1016/j. drudis2008.07.005.

Grosdidier A, Zoete V, Michielin O. 2011. SwissDock, a protein-small molecule docking web service based on EADock DSS. Nucl. Acids Res. 39: W270-W277. DOI: 10.1093/nar/gkr366.

Hess B, Kutzner C, van der Spoel D, Lindahl E. 2008. GROMACS 4: Algorithms for highly efficient, load-balanced, and scalable molecular simulation. J. Chem. Theor. Comput. 4: 435-447. DOI: 10.1021/ ct700301q.

Hildmann C, Ninkovic M, Dietrich R, Wegener D, Riester D, Zimmermann T, Birch OM, Bernegger C, Loidl P, Schwienhorst A. 2004. A new amidohydrolase from Bordetella or Alcaligenes strain FB188 with similarities to histone deacetylases. J. Bacteriol. 186: 2328-2339. DOI: 10.1128/JB.186.8.2328-2339.2004.

Hildmann C, Wegener D, Riester D, Hempel R, Schober A, Merana J, Giurato L, Guccione S, Nielsen TK, Ficner R, Schwienhorst A.
2006. Substrate and inhibitor specificity of class 1 and class 2 histone deacetylases. J. Biotechnol. 124: 258-270. DOI: 10.1016/ j.jbiotec.2006.01.030.

Jorgensen WL, Madura JD. 1985. Temperature and size dependence for Monte Carlo simulations of TIP4P water. Mol. Phys. 56: 1381-1392. DOI: 10.1080/00268978500103111.

Kawasaki Y, Freire E. 2011. Finding a better path to drug selectivity. Drug Discov. Today 16: 985-990. DOI: 10.1016/j.drudis.2011.07.010.

Ladbury JE, Klebe G, Freire E. 2009. Adding calorimetric data to decision making in lead discovery: a hot tip. Nat. Rev. Drug Discov. 9: 23-27. DOI: $10.1038 / \mathrm{nrd} 3054$.

Marks PA. 2010. Histone deacetylase inhibitors: a chemical genetics approach to understanding cellular functions. Biochim. Biophys. Acta 1799: 717-725. DOI: 10.1016/j.bbagrm.2010.05.008.

Neumann L, Von König K, Ullmann D. 2011. HTS reporter displacement assay for fragment screening and fragment evolution toward leads with optimized binding kinetics, binding selectivity, and thermodynamic signature. Methods Enzymol. 493: 299. DOI: 10.1016/B9780-12-381274-2.00012-1.

Nielsen TK, Hildmann C, Dickmanns A, Schwienhorst A, Ficner R. 2005. Crystal structure of a bacterial class 2 histone deacetylase homologue. J. Mol. Biol. 354: 107-120. DOI: 10.1016/j.jmb.2005.09.065.

Ohtaka H, Freire E. 2005. Adaptive inhibitors of the HIV-1 protease. Prog. Biophys. Mol. Biol. 88: 193-208.

Paris M, Porcelloni M, Binaschi M, Fattori D. 2008. Histone deacetylase inhibitors: from bench to clinic. J. Med. Chem. 51: 1505-1529. DOI: 10.1021/jm7011408.

Peng L, Seto E. 2011. Deacetylation of nonhistone proteins by HDACs and the implications in cancer. Handb. Exp. Pharmacol. 206: 39-56. http://www.hubmed.org/display.cgi?uids=21879445. DOI: 10.1007/ 978-3-642-21631-2 3.

Riester D, Hildmann C, Schwienhorst A, Meyer-Almes FJ. 2007. Histone deacetylase inhibitor assay based on fluorescence resonance energy transfer. Anal. Biochem. 362: 136-141. DOl: 10.1016/j.ab.2006.12.019.

Riester D, Hildmann C, Haus P, Galetovic A, Schober A, Schwienhorst A, Meyer-Almes FJ. 2009. Non-isotopic dual parameter competition 
assay suitable for high-throughput screening of histone deacetylases Bioorg. Med. Chem. Lett. 19: 3651-3656. DOI: 10.1016/j.bmcl.2009. 04.102.

Ropero S, Esteller M. 2007. The role of histone deacetylases (HDACs) in human cancer. Mol. Oncol. 1: 19-25. DOI: 10.1016/j.molonc.2007.01.001.

Ruben AJ, Kiso Y, Freire E. 2006. Overcoming roadblocks in lead optimization: a thermodynamic perspective. Chem. Biol. Drug Des. 67: 2-4. DOI: $10.1111 / \mathrm{j} .1747-0285.2005 .00314 . x$.

Shankar S, Srivastava RK. 2008. Histone deacetylase inhibitors: mechanisms and clinical significance in cancer: HDAC inhibitor-induced apoptosis. Adv. Exp. Med. Biol. 615: 261-298. DOI: 10.1007/978-1-4020-6554-5_13.

Shrake A, Rupley JA. 1973. Environment and exposure to solvent of protein atoms. Lysozyme and insulin. J. Mol. Biol. 79: 351-371. DOl: 10.1016/0022-2836(73)90011-9.

The clinical trials website. http://clinicaltrials.gov/ [22 August 2013]

Wegener D, Hildmann C, Riester D, Schwienhorst A. 2003. Improved fluorogenic histone deacetylase assay for high-throughputscreening applications. Anal. Biochem. 321: 202-208. DOI: 10.1016/ S0003-2697(03)00426-3.
Williams DH, Stephens E, Zhou M. 2003. Ligand binding energy and catalytic efficiency from improved packing within receptors and enzymes. J. Mol. Biol. 329: 389-399. DOI: 10.1016/S0022-2836(03) 00428-5.

Zhang J, Chung TDY, Oldenburg KR. 1999. Validation of High Throughput Screening Assays. J. Biomol. Screen. 4. DOI: 10.1177/ 108705719900400206.

Zhang Y, Feng J, Jia Y, Wang X, Zhang L, Liu C, Fang H, Xu W. 2011. Development of tetrahydroisoquinoline-based hydroxamic acid derivatives: potent histone deacetylase inhibitors with marked in vitro and in vivo antitumor activities. J. Med. Chem. 54: 2823. DOI: $10.1021 / \mathrm{jm} 101605 z$.

\section{SUPPORTING INFORMATION}

Additional supporting information may be found in the online version of this article at the publisher's web site. 